\title{
A Gaijin-like miniature inverted repeat transposable element is mobilized in rice during cell differentiation
}

\author{
Hai-Tao Dong ${ }^{1}$, Lu Zhang ${ }^{1}$, Kang-Le Zheng ${ }^{2}$, Hai-Gen Yao ${ }^{3}$, Jack Chen ${ }^{4}$, Feng-Chi Y ${ }^{3}$, Xiao-Xing Yu', \\ Bi-Zeng Mao', Dong Zhao', Jian Yao ${ }^{3}$ and De-Bao Li ${ }^{1 *}$
}

\begin{abstract}
Background: Miniature inverted repeat transposable element (MITE) is one type of transposable element (TE), which is largely found in eukaryotic genomes and involved in a wide variety of biological events. However, only few MITEs were proved to be currently active and their physiological function remains largely unknown.

Results: We found that the amplicon discrepancy of a gene locus LOC_Os01g0420 in different rice cultivar genomes was resulted from the existence of a member of Gaijin-like MITEs (mGing). This result indicated that mGing transposition was occurred at this gene locus. By using a modified transposon display (TD) analysis, the active transpositions of $m$ Ging were detected in rice Jiahua No. 1 genome under three conditions: in seedlings germinated from the seeds received a high dose $\gamma$-ray irradiation, in plantlets regenerated from anther-derived calli and from scutellum-derived calli, and were confirmed by PCR validation and sequencing. Sequence analysis revealed that single nucleotide polymorphisms (SNPs) or short additional DNA sequences at transposition sites post mGing transposition. It suggested that sequence modification was possibly taken place during mGing transposition. Furthermore, cell re-differentiation experiment showed that active transpositions of both $m$ Ging and $m$ Ping (another well studied MITE) were identified only in regenerated plantlets.
\end{abstract}

Conclusions: It is for the first time that $m$ Ging active transposition was demonstrated under $\boldsymbol{\gamma}$-ray irradiation or in cell re-differentiation process in rice. This newly identified active MITE will provide a foundation for further analysis of the roles of MITEs in biological process.

\section{Background}

Transposable elements (TEs) are DNA fragments that are able to translocate into other positions in the genome. About six decades ago, TEs were discovered in maize as the genetic agents that are responsible for the pigment alteration on mutant kernels by Barbara McClintock [1]. Large amount of TEs are found in eukaryotic genomes and involved in a wide variety of biological events including viral integration and replication and dispersal of antibiotic resistant genes [2]. Amongst all the different types of TEs, miniature inverted repeat transposable elements (MITEs) have been exploited as an effective and informative genetic

\footnotetext{
* Correspondence: debl3@yahoo.com

'Institute of Biotechnology, College of Agriculture and Biotechnology,

Zhejiang University, Hangzhou 310029, China Full list of author information is available at the end of the article
}

tool for plant genome analyses [3]. MITEs resemble typical non-autonomous DNA transposons, which have a small size of less than 500 base pair (bp), terminal inverted repeats (TIRs) and target site duplications (TSDs). But high copy number (up to 10000 copies per family), size homogeneity and preference of insertion into single-copy regions are the additional characteristics that distinguish MITEs from other non-autonomous DNA transposons $[4,5]$. Unlike autonomous DNA transposons, non-autonomous DNA transposons required transposases encoded by autonomous elements for transposition and often found to be the autonomous elements internal deletion derivatives that the terminal binding sites are preserved for transposase interaction [6]. Studies showed that the sequences of some MITE families were very similar to autonomous DNA transposons, suggesting that these MITEs were originally from
C Biomed Central 
autonomous transposons and be able to mobilize by the use of transposases encoded from the corresponding autonomous transposons [5,7]. Sometimes, it is difficult to directly correlate a given MITE family with an autonomous transposon present within the same genome, especially when the sequence similarity between MITEs and the closest autonomous element is restricted to the TIRs [8-10].

In plant, most of the MITEs are classified into two groups: Tourist-like MITEs and Stowaway-like MITEs based on shared TIRs and TSD sequences [11]. The Tourist-like rice element mPing was identified as the first actively transposing MITE. It was primarily found actively transposed in long-term cell cultures, newly derived anther cultures and plants derived from seeds with $\gamma$-ray irradiation [12-14]. Sequence homology and co-transposition analysis in Arabidopsis revealed that the transposases encoded by two members of the PIF/ IS5 superfamily, Pong and Ping, were essential for the mobilization of mPing [15]. Recently, a study demonstrated that the Stowaway-like MITEs could also be actively transposed in vitro in a yeast co-transposition system with rice autonomous Mariner-like transposons [16]. The association of MITEs and autonomous elements in both Tourist-like and Stowaway-like elements, high copy number, and the great variety of MITEs in the genomes are the indications that the number of active MITEs may not be as few as previously anticipated.

Gaijin-like MITEs, a sub-class of Tourist-like MITE, were originally discovered in 1996 and computationally proved to be mobilized at some stages of rice evolution [17]. The current status of Gaijin-like MITEs in rice and their associated biological significance have not yet been investigated. Our investigation showed for the first time that members of Gaijin family (named mGings) are actively transposing in seedlings of rice cultivar Jiahua No. 1 germinated from the seeds after irradiated by $\gamma$ ray. Using different culture conditions, we also detected active transpositions of $m$ Gings in plantlets regenerated from anther-derived calli. These results revealed that $m$ Gings are currently active and further investigation of $m$ Ging transposition would provide a foundation for an in-depth analysis of the roles of MITEs in biological processes and evolution in rice.

\section{Results}

\section{A Gaijin-like MITE conserved after intron retention}

Intron retention is one basic mode of alternative premRNA splicing in plants and eukaryotic cells. This mRNA transcription mechanism plays an important role in genetic regulation and provides evolutionary flexibility by manipulating the protein variability and diversity from the genome [18]. In analysis of a putative leucine- rich repeat protein $(P L R R P)$ (Locus Name: LOC_Os01g04720) using the rice genome browser http://rice.plantbiology.msu.edu from Rice Genome Annotation Project - Michigan State University [19], we found that two mRNA transcripts in this expressed protein and their size differences were due to the 4th and 8 th introns retention. To investigate the discrepancy of the PLRRP spliced transcript variants, two rice cultivars, Xiushui 11 and Zhonghua 11, were selected for analysis. RT-PCR analysis showed that two amplicons were amplified from the 4th intron of the PLRRP mRNA transcripts in the both cultivars (Figure 1a). The sizes of amplicons were consistent in different organs; however, the size of a higher molecular weight amplicon was different between the two cultivars. Similar amplicons were detected in the PCR amplification from the genomic DNAs (gDNAs) of the two rice cultivars (Figure 1b). Sequence analysis of all the aforementioned amplicons revealed that an ATTAATAT sequence and a fragment with the length of $146 \mathrm{bp}$ were contributed to the amplicons size increment, and the size increment in Xiushui 11 was inherited from chromosomal transcription (Figure 1c). Sequence homology search and structural examination of the $146 \mathrm{bp}$ fragment sequence showed that about 1,000 highly aligned sequences were found in the NCBI rice genome database and short TIRs as well as a terminal ATA duplication (Figure 1d) were identified at both ends of the element. The presence of high copy number and structural features suggested that the $146 \mathrm{bp}$ fragment was a MITE. Further analysis of the $146 \mathrm{bp}$ fragment with the CENSOR program against Repbase [20] showed that it shared 92\% homology with a previously reported MITE Gaijin (Figure 1e). The sequence homology and MITE features denoted that this 146 bp fragment is a member of Gaijin family, and we designated it as miniature Ging (mGing) element.

The identification of $m$ Ging in the 4 th intron of PLRRP clarified the higher molecular weight of mRNA transcripts in Xiushui 11 compared to Zhonghua 11. As shown in Figure 1e, the $m$ Ging in the 4th intron of Xiushui 11 did not transcribed into the normal transcript but mGing was transcribed into the transcript variant due to the intron retention. The insertion of $m$ Ging and together with intron retention at the $4^{\text {th }}$ intron could explain the size inconsistency of the spliced variants detected between different rice strains. We suggest that the transposition of $m$ Ging, as an active MITE, might contribute to the modification of gene expression if $m$ Ging transposes into a promoter region or it affects a regulatory element of the gene. For example, $m$ Ging transposition plays a role in the sequence coding of a functional alternative-splicing product. 


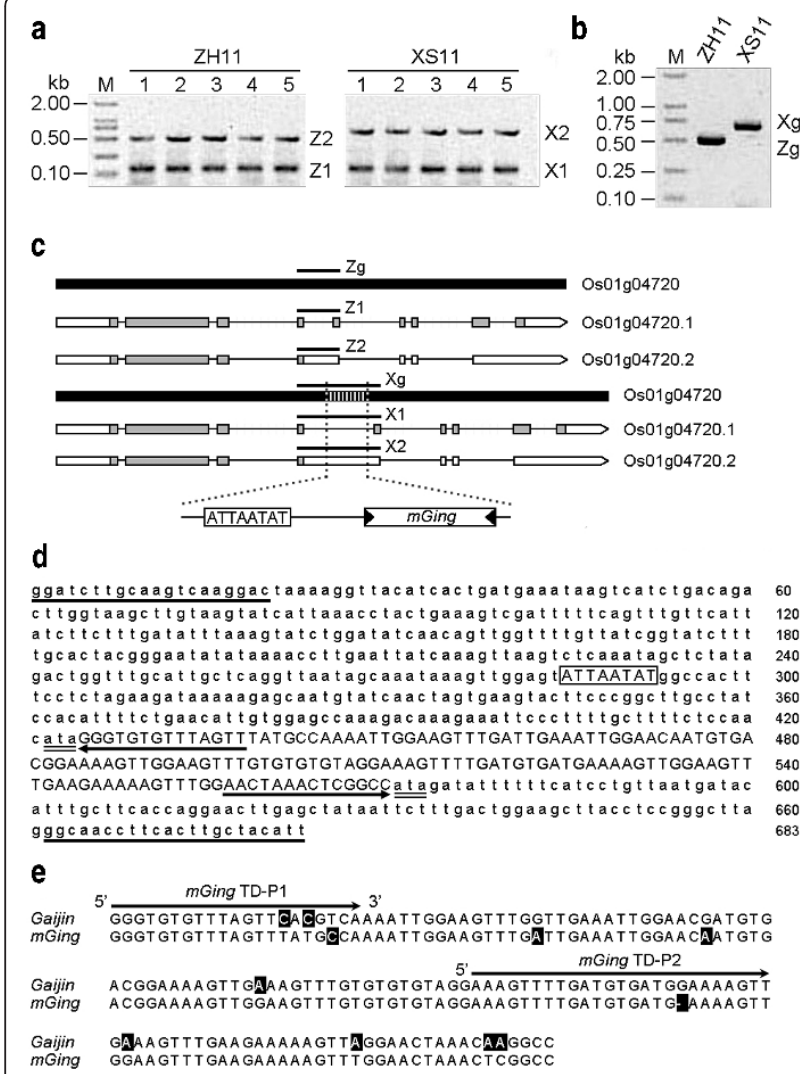

Figure 1 Discovery of a Gaijin-like MITE in the 4th intron of PLRRP gene. (a) Expression profile of PLRRP gene in different organs of two rice cultivars, Zhonghua 11 and Xiushui 11. RNA samples were extracted from callus (lane 1), root (lane 2), stem (lane 3), leaf (lane 4) and embryo (lane 5) and amplified by the AS primer pair. The size of the lower molecular weight RT-PCR amplicons of Zhonghua 11 (Z1) and Xiushui 11 (X1) were similar. However, the size of the higher molecular weight amplicons in Xiushui 11 (X2) was bigger than Zhonghua 11 (Z2). (b) DNA polymorphism of PLRRP gene. gDNAs were extracted from rice cultivars and amplified by the AS primer pair. The molecular weight of PCR amplicon from Zhonghua 11 (Zg) was smaller than Xiushui 11 (Xg). (c) PLRRP gene and its spliced mRNA transcripts. PLRRP gene (Zg, thick black lines) was composed of 9 exons (white and grey boxes) and 8 introns (thin black lines). The additional fragment at the 4th intron was indicated with vertical strip lines and the two additional fragments, $8 \mathrm{bp}$ ATTAATAT fragment and a $146 \mathrm{bp}$ fragment (mGing) were illustrated in the pictograph. Amplicons of Zhonghua 11 (Z1, Z2 and $\mathrm{Zg}$ ) and Xiushui 11 (X1,X2 and $\mathrm{Xg}$ ) from part (a) and part (b) were indicated. (d) Sequence analysis of PLRRP gene in Xiushui 11. Underlines showed the AS primer pair sequences. Doubleunderlines and arrows indicated the ATA duplication and the TIRS, respectively. The position of ATTAATAT sequence was boxed. Capital letters revealed the $146 \mathrm{bp}$ fragment. (e) Sequence alignment of mGing against Gaijin. The Gaijin sequence was obtained from Repbase. The alignment was made using the online ClustalW program with the different residue at each position highlighted. The arrows indicated the $m$ Ging TD primers.

\section{Copy number of $m$ Ging in rice cultivars and wild rice accessions}

To determine the copy number of $m$ Ging in rice genome, sequence homology search was performed with whole genome sequences of two rice cultivars, Nipponbare and 93-11 due to the availability of full genome sequences, and Arabidopsis genome sequences were used as a control. More than 3,000 high-scoring segment pairs (HSPs) matching to $m$ Ging were identified in the genomes of two rice cultivars, while no HSPs were detected in Arabidopsis genome (See additional file 1: sequence homology search for $m$ Ging copy numbers). To determine the chromosomal distribution of mGing in Nipponbare genome, 1,055 high-matching HSPs (Nipponbare genome) with e-values less than 1e-20 from the sequence alignment report were mapped onto 12 chromosomes. As indicated by Figure 2a, mGings were abundant in euchromatin, the form of chromosomal material that contains a majority of active genes. In addition, GC contents of these 1055 HSPs were calculated to see if mGing preferably located in an AT-rich region. Compared to the randomly picked sequences with a GC content around $43 \%$, the sequences close to the mGing locations only have a lower GC content, especially the $100 \mathrm{bp}$ upstream and downstream of the mGing location (Figure 2b). A pair of TIR with the length of $15 \mathrm{bp}$ was identified from the composition analyses of the $18 \mathrm{bp}$ sequence at the $5^{\prime}$ and 3 ' terminal of 1055 high-matching HSPs. Base composition analysis of TIRs indicated that two TIR sequences were not perfectly conserved among mGing copies. Bases at the third and fourth position "GT" in the 5'TIR, for example, were not complementary with their counterparts "GG" in the 3'TIR (Figure 2c).

Southern blot analysis was initially used to qualitatively examine the $m$ Ging copy number in rice genome. In agreement with the results obtained by sequence homology search, signals detected from hybridization with the ${ }^{33} \mathrm{P}$ labeled $m$ Ging specific probe showed a strong smear (See additional file 2: Southern blot analysis of $m$ Ging), indicating the high copy number of mGing in rice genome. A modified transposon display (TD, see Methods) analysis was then used to identify the copy number of $m$ Ging, and mPing was used to evaluate the feasibility of the TD analysis with its specific primers (See additional file 3: Polymorphism of locations of $m$ Ging (a) and mPing (b) in rice genomes) [12]. Compared with the high numbers of fragments observed in mGing TD analysis, mPing had fewer amplicons but appeared to be much more polymorphic. To confirm that the bands amplified by TD were linked with mGing, 15 bands 


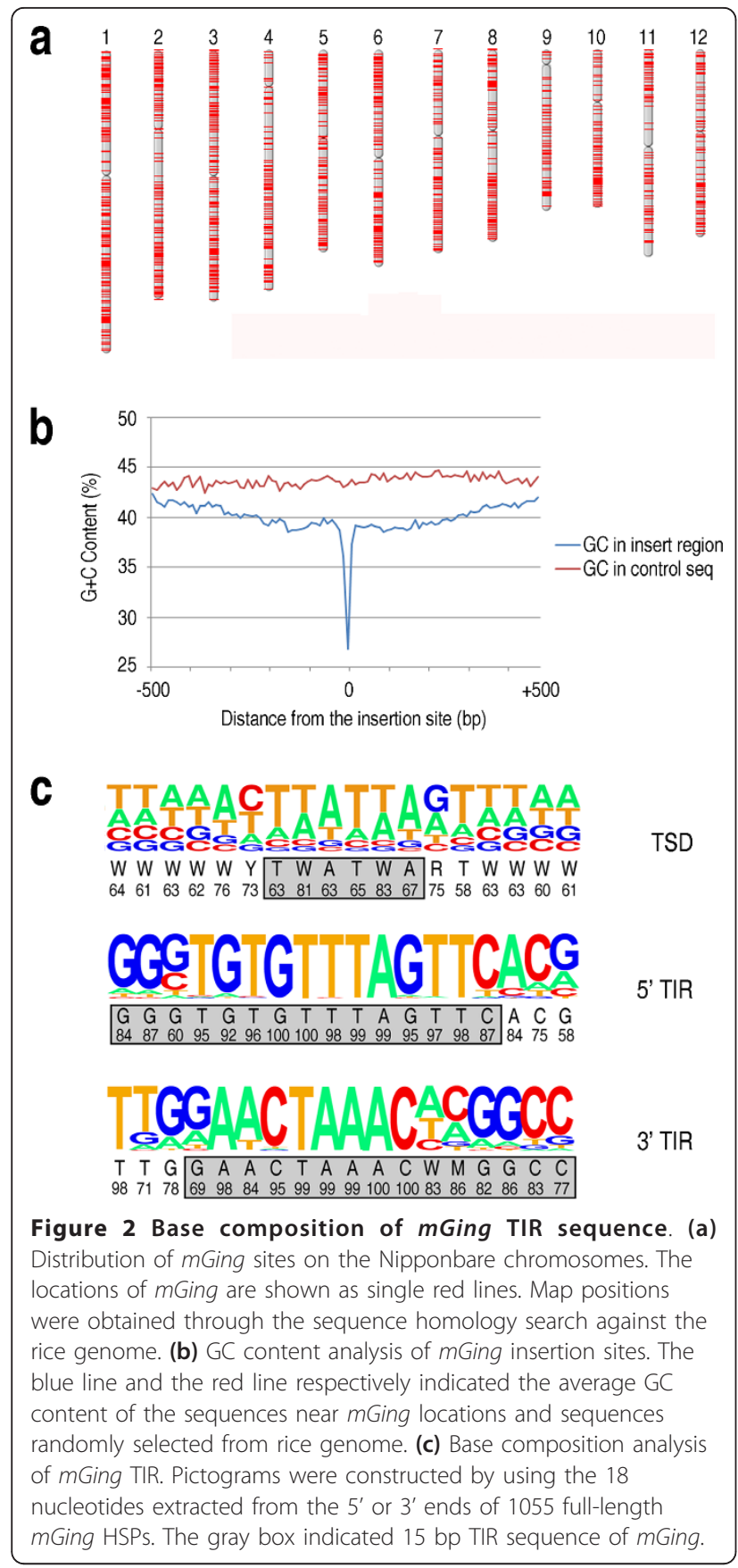

from TD gel (additional file 3a, PstI, line 1) were recovered, reamplified, cloned and sequenced. The sequences of all bands contained the partial sequences of $m$ Ging, confirming that all bands were $m$ Ging-anchored amplicons (See additional file 4: Sequence alignment of 15 bands from TD gel).

In addition, a series of $O$. rufipogon accessions from different planting regions and in cultivars of $O$. sativa subspecies were used to examine the copy number of mGing by TD analysis (Figure 3 ). The band

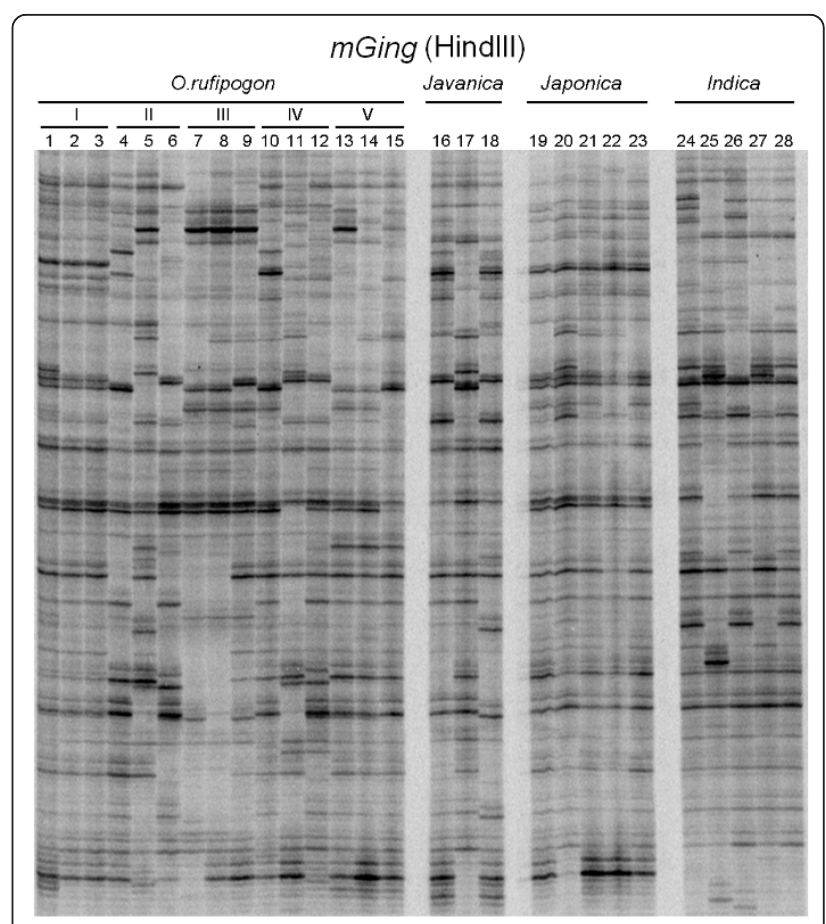

Figure 3 TD analysis of $m$ Ging in various rice cultivars and wild rice accessions. The gDNA samples were digested with HindIII and ligated to the HindIII cassettes for TD analysis. A collection of fifteen O. rufipogon accessions, including three lines from Guangdong province of China (1, S7943; 2, S7944; 3, S6238), three accessions from Guangxi province of China $(4,7023 ; 5, G X 4 ; 6, G X 6)$, three accessions from Fujian province of China $(7,7045 ; 8,7074 ; 9,7075)$, three accessions $s$ from Hubei province of China (10, Wuye No. 1; 11, Wuye No. 2; 12, Wuye No. 3) and three accessions from Jiangxi province of China (13, 7028; 14, 7029; 15, 550-1), three javanica cultivars (lane 16 to 18: Aus 114, Jaya and Dular), five japonica cultivars (lane 19 to 23: 19, Nipponbare; 20, Yueguang; 21, Liming; 22, Nongken 58; 23,Qiuguang) and five indica cultivars (lane 24 to 28: IR 36, Tetep, Milyang 46, IR 24 and IR 30, respectively) were randomly selected.

polymorphism of $m$ Ging in different accessions of $O$. rufipogon suggested that the transpositions of $m$ Ging were potentially occurred before rice domestication. TD analysis also showed that the banding patterns of mGing among O. rufipogon plants coming from the same geographic planting region were not consistent, indicating that $m$ Ging were possibly mobilized under individual natural circumstance. The similarity of the TD banding patterns of mGing among japonica cultivars was significantly higher than that among javanica or indica cultivars, signifying that $m$ Ging had been mobilized since rice domestication and might be an active MITE. The principal component analysis (PCA) also revealed significant variation among the samples (See additional file 5 : Principal component analysis clustering of 13 rice cultivars and 18 wild rice accessions). The band patterns of most $O$. rufipogon accessions were not clustered by their 
regions, except the group III. The band profiles of indica cultivars were well clustered by their sub-species, whereas only 3 of 5 japonica cultivars were grouped together.

\section{$\gamma$-ray irradiation induced $m$ Ging transposition}

To test the current mobilization of $m$ Ging, its transposition in stress conditions, including $\gamma$-ray irradiation and culture experiments, was examined by the aid of TD. Rice cultivar Jiahua No. 1 (japonica) was chosen as the plant material for both $\gamma$-ray irradiation and the following anther culture experiments due to its relatively high rates of callus induction and re-differentiation in culture experiments. In $\gamma$-ray irradiation experiment, one inbred tiller of a single Jiahua No. 1 plant was chosen to make sure the consistence of genetic background. Mature seeds harvested from this single tiller were divided into three groups and then received $\gamma$-ray irradiation for 30 minutes at different dosage. Seeds without irradiation were $100 \%$ germinated, while $90 \%$ of seeds were germinated after irradiation at $300 \mathrm{~Gy}$ and $40 \%$ at 500 Gy. As shown in Figure 4, the polymorphic bands of mGing were observed among seedlings irradiated at 500 Gy ( $m$ Ging, group III), but not in seedlings without irradiation (mGing, group I) or irradiated at 300 Gy (mGing, group II). Band polymorphism was not observed in the TD control experiment, where the specific TD primers of another MITE, $I D-1$, were used with the same gDNA samples from seedlings irradiated at 500 Gy (ID-1, group III). These results suggested that the mGing might be induced to actively transpose by $\gamma$-ray irradiation and the observed polymorphic bands of mGing were less likely due to genome rearrangement, DNA breakage or sample cross-contamination.

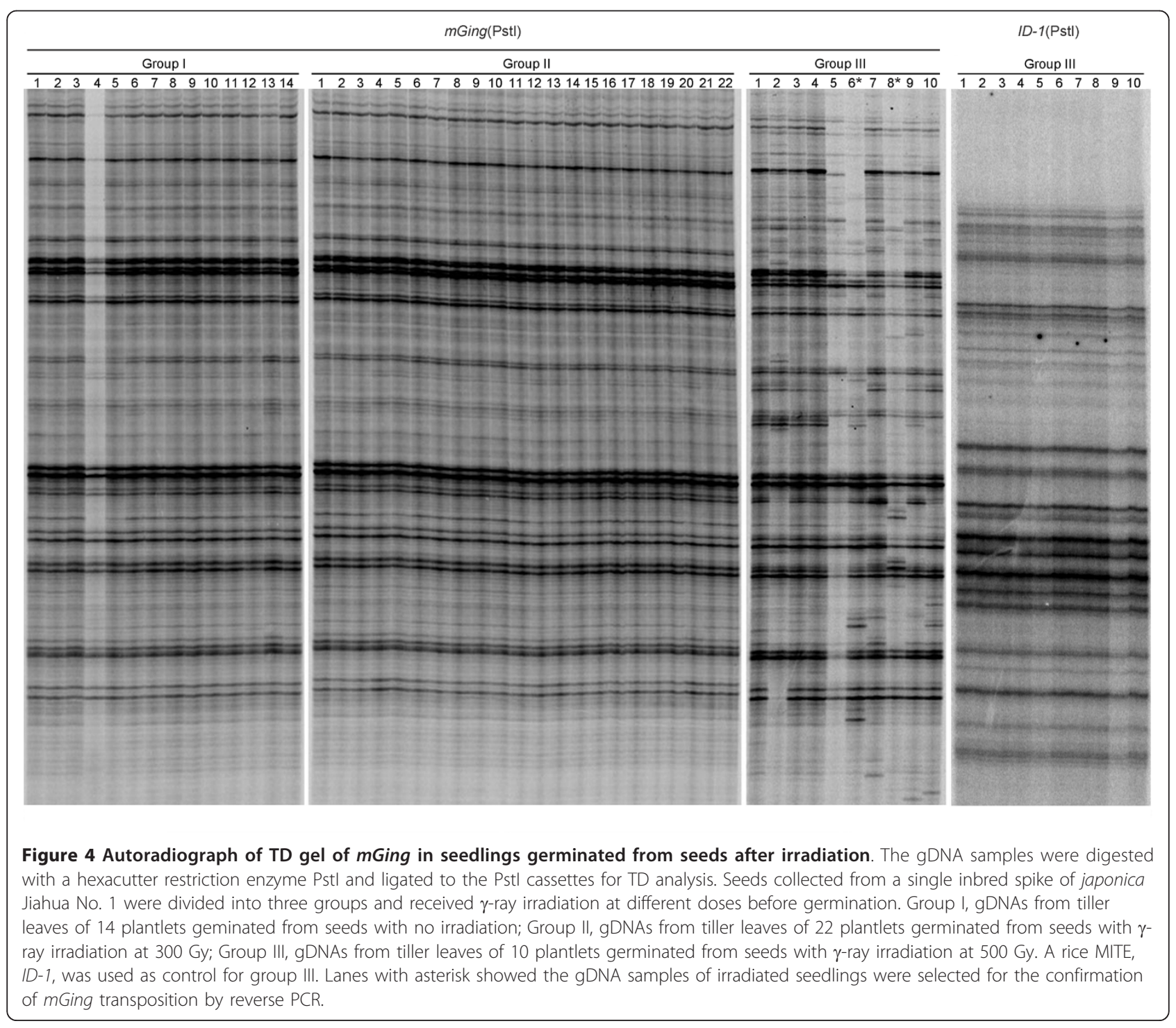


Whether the transposition of mGing was induced by $\gamma$-ray irradiation was also examined by PCR validation experiment. The inverse PCR amplification of DNA fragments using gDNAs from 2 irradiated seedlings (Figure 4, mGing, group III, lane 6 and 8) and mGing TD primers was firstly performed to locate the genomic sequence disrupted by $m$ Ging. Amplicons were cloned and totally 479 clones were selected for sequencing. Sequence analyses showed that 55 fragments were unique $m$ Ging-anchored sequences. Due to the unavailability of the whole genome sequence of cultivar Jiahua No.1, sequence homology search with 55 fragments as queries was performed against the reference genome sequence (Nipponbare). After the exclusion of mGinganchored fragments with short length or numerous HSPs, 21 unique flanking sequences were identified as templates for PCR primer designation. The gDNAs of ten seedlings irradiated with 500 Gy dose $\gamma$-ray, in which polymorphic bands of mGing were observed by TD assay (Figure 4, group III), were chosen as samples for PCR validation experiment. Two bands of different size were amplified from three genomic loci (Figure 5a). Sequence analyses showed that the bands of higher molecular weight were mainly resulted from the presence of $m$ Ging (Figure $5 \mathrm{~b})$. These data indicated that $\gamma$ ray irradiation induced $m$ Ging transposition in rice. In addition, the high frequency of "double band" observed at each locus in ten samples suggested the contribution from high dose irradiation.

\section{Mobilization of $m$ Ging during tissue culture processes}

As high dose irradiation usually causes genome instability, the transposition of $m$ Ging was further examined in anther culture condition where no irradiation was involved. Initially, the gDNAs of 10 randomly selected paddy plants were extracted for TD assay. Band polymorphism of the $m$ Ging was identified among individual plants within Jiahua No.1 (Figure 6a, group I). Thus, only a single plant was selected for the following investigations (Figure 6a, group I, lane 1). Anthers containing microspores at the midunicleate stage from a spike of the single plant were inoculated into 22 test tubes containing the one-step cell culture medium. After eight weeks, 14 green plantlets were regenerated from calli in four of the test tubes, but calli re-differentiation was not observed in the remaining 18 tubes. The gDNAs were extracted from 14 green plantlets (Figure 6a, group III) and 14 calli randomly selected from the 18 tubes (Figure $6 a$, group II). Polymorphic bands were observed in half of the plantlets regenerated from anther-derived calli, indicating that the transposition of mGing might be induced by anther culture (Figure 6a, group III).

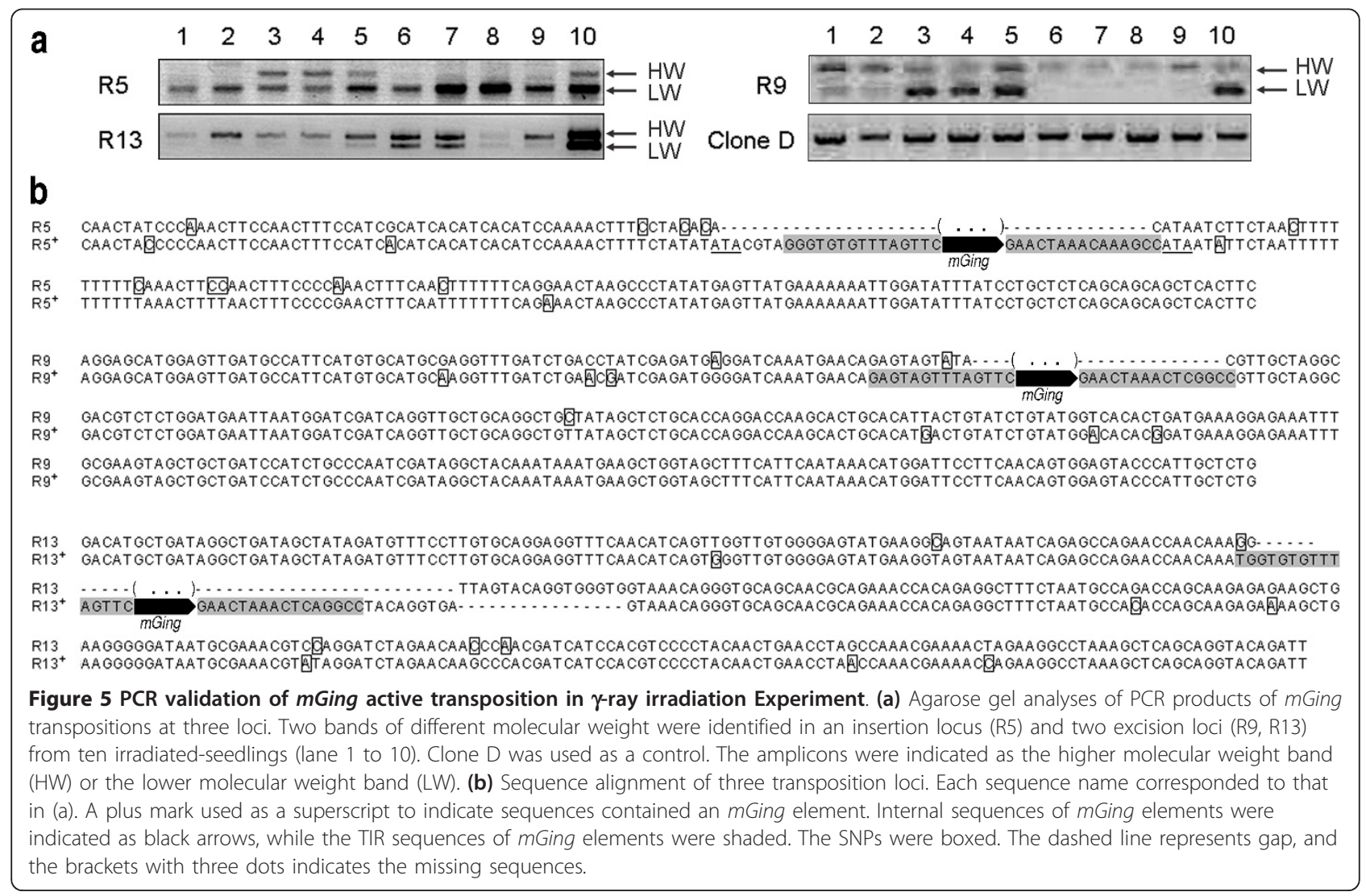




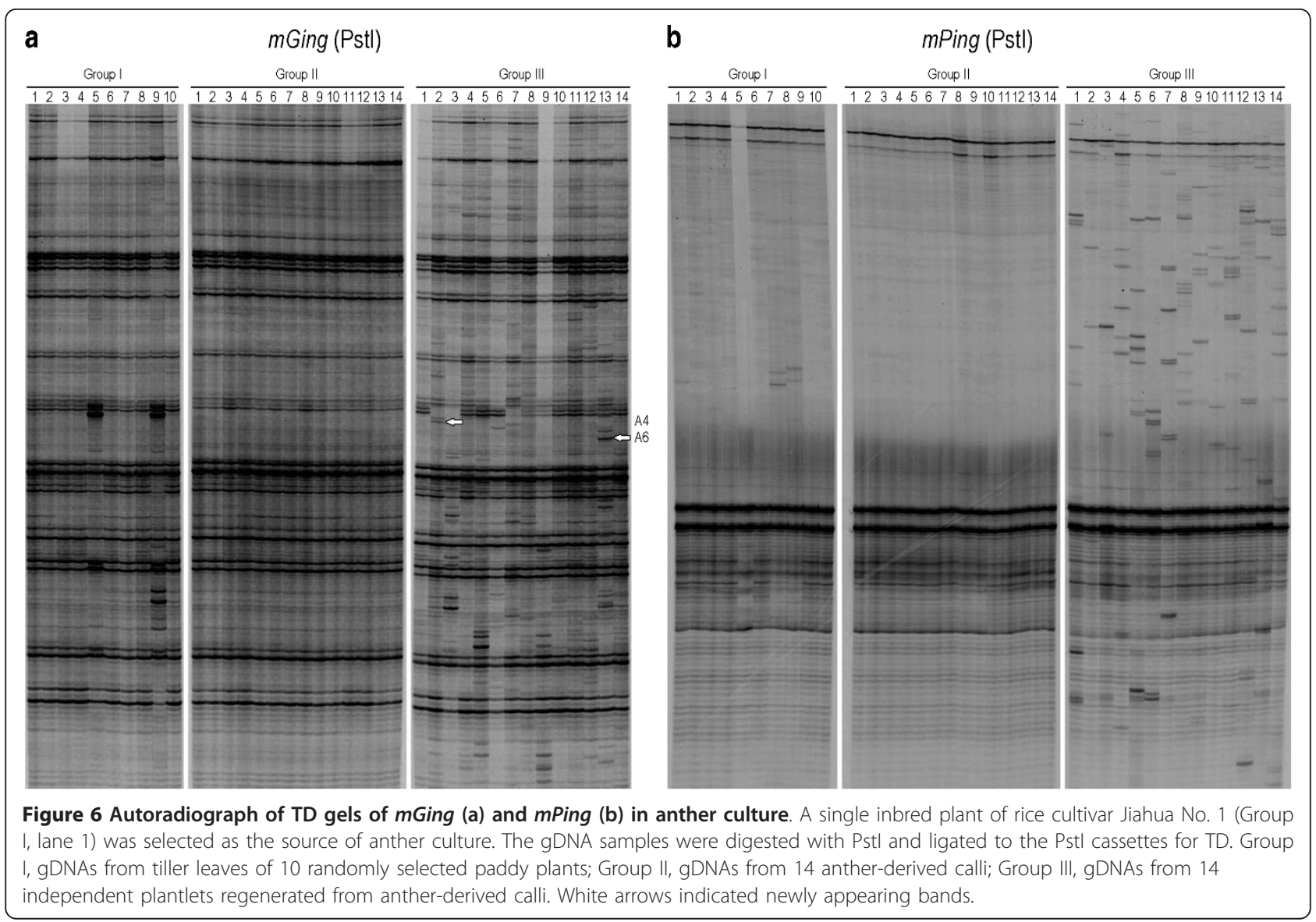

To investigate the band polymorphism observed in the above TD experiments was a result of $m$ Ging transposition, 8 random polymorphic bands from TD gels (Figure 6a, group III) were excised, cloned and identified to be $m$ Ging-anchored amplicons. PCR validation experiments with primers designed on the basis of flanking sequences of the 8 polymorphic bands were then carried out to confirm the active mGing transposition in the genome of 14 regenerated plantlets. Results showed that two bands of different size were amplified from 2 out of the 8 primer pairs and sequence analysis confirmed that $m$ Ging element was contributed to the size increment of the amplicons at higher molecular weight (Figure 7a). Because of the consistence of genetic backgrounds of the 14 samples (all were regenerated from anthers of a single inbred spike), the amplified larger amplicons were the results of $m$ Ging insertions at the corresponding genome loci. These results demonstrated that mGing active transposition was induced by anther culture condition.

Moreover, the transposition frequencies of mGing/ mPing were examined in scutellum-derived calli and regenerated plantlets to investigate if their transpositions were influenced by the explants (starting materials used for tissue culture). TD analysis revealed that mPing exhibited a similar profile as noted in anther cultures, while only a small amount of polymorphic bands of $m$ Ging were detected in scutellum-derived plantlets (See additional file 6: Autoradiograph of TD gels of $m$ Ging (a) and mPing (b) in embryogenic culture). This result indicated that mGing transposition may be associated with explants and its transposition frequency is significantly lower than mPing in scutellum-derived plantlets.

\section{Sequence specificities of the mGing transposition site}

As shown in Figure 5 and 7, PCR validations of $\gamma$-ray irradiation and cell culture experiments totally detected the five active $m$ Ging copies and identified their transposition sites. Sequence variations at the surrounding regions of mGing elements were discovered from sequencing of the three insertions (Figure 5b, R5; Figure 7b, A4, A6) and two excisions (Figure 5b, R9, R13). For example, examination of the three insertions showed additional short DNA (Figure 5b, R5; Figure 7b, A4) or TSD (Figure 7b, A6) at either 5' and/or 3' end of the complete $m$ Ging sequence after insertion into the new sites. Analysis of the two excision sequences identified the imprecise excision (Figure 5b, R9) or additional 


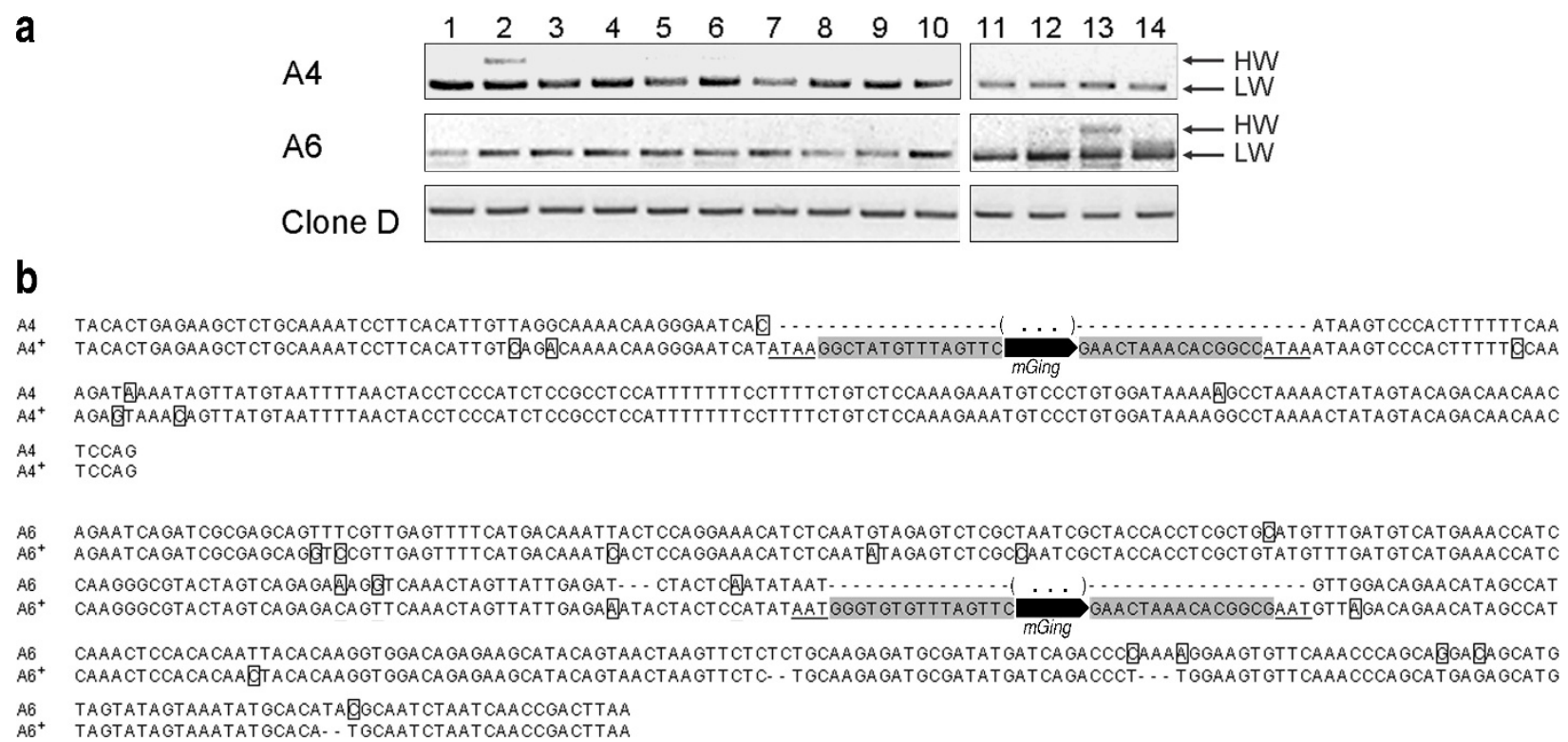

Figure 7 PCR validation of $m$ Ging active transposition in anther culture experiment. (a) Agarose gel analyses of PCR products of $m G i n g$ transposition loci in anther culture experiment. Two bands of different molecular weight were identified in two new transposition loci (A4 and A6) from 14 plantlets regenerated from anther-derived calli (lane 1 to 14). (b) Sequence alignment of two transposition loci. Each sequence name corresponded to that in (a). Clone D was used as a control in both experiments. The amplicons were indicated as the higher molecular weight band (HW) or the lower molecular weight band (LW). A plus mark was used to identify the sequence with an mGing element. The dashed line represents gap and the brackets with three dots indicates the missing sequences. The TIRs of mGing elements were shaded, the TSDs were underlined, and the SNPs were boxed.

short sequences (Figure 5B, R13) at the mGing excision sites. All transposition sites contained scattered single nucleotide polymorphism (SNPs) in the flanking sequences at both ends of the mGing elements. These sequences variations were possible explanations that only 2 out of 8 polymorphic bands excised from TD gel in anther culture experiment were confirmed by the PCR validation, and indicated that active transposition of $m$ Ging potentially associated with the introduction of chromosomal changes at the flanking regions. Furthermore, sequence comparison also identified the polymorphism among the five active mGing copies, the sizes of which were varied from 143 to $148 \mathrm{bp}$ (See additional file 7: Sequence alignment of the $m$ Ging elements from new transposition sites). However, sequence conservation between any two active copies was still at a high level (more than 90\% matched with each other).

\section{Correlation of $m$ Ging transposition and re-differentiation}

We noted that no case of mGing transposition was detected in anther-derived or scutellum-derived calli (Figure 6a, group II; additional file 6a, group II). Similar profile was also observed when TD analysis was carried out using the above materials with mPing specific primers (Figure 6b, additional file 6b, group II). To investigate if the transposition of mGing or mPing was more easily detected in regenerated plantlets, two experimental systems were designed to examine the transposition frequency of $m$ Ging/mPing in calli.

In the first experimental system, 20 anther-derived callus samples yielded from four callus pieces were used to detect the transposition of mGing or mPing (see material and methods). TD analysis showed that no active transposition of $m$ Ging or mPing was detected in those 20 samples, suggesting that it is difficult to detect transposition of mGing or mPing in callus during cell proliferation (Figure 8a). In the second experimental system, we used 18 anther-derived calli, each three of which were from one independent anther-derived callus and respectively cultured in three different culturing conditions (see material and methods). Compared with the fresh calli in group I, local callus cells in group II appeared to be green, while morphological changes, such as browning appearance, local cell death or rootlike structures, were observed on calli in group III (Figure $8 \mathrm{~b})$. The transpositions of $m$ Ging/mPing were examined by TD analysis using gDNAs extracted from all of the calli. The amplified band number of mGing was much higher than that of mPing, which made it difficult for the polymorphic bands to be visible in mGing TD assay. In contrast, polymorphic bands of mPing were obviously detected in group II and III. These results further confirmed the association of callus re-differentiation with active transpositions of $m$ Ging/mPing. 


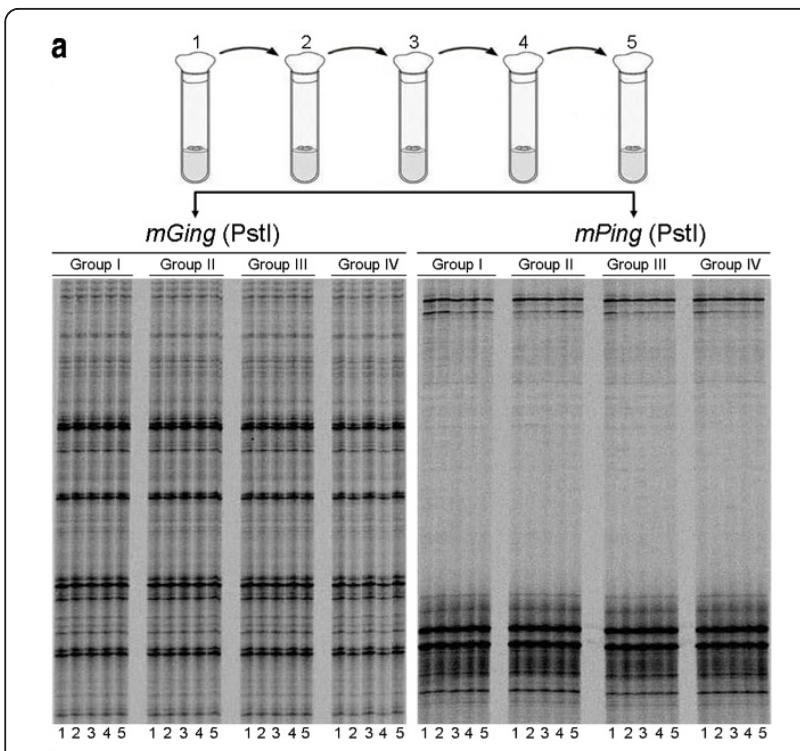

b
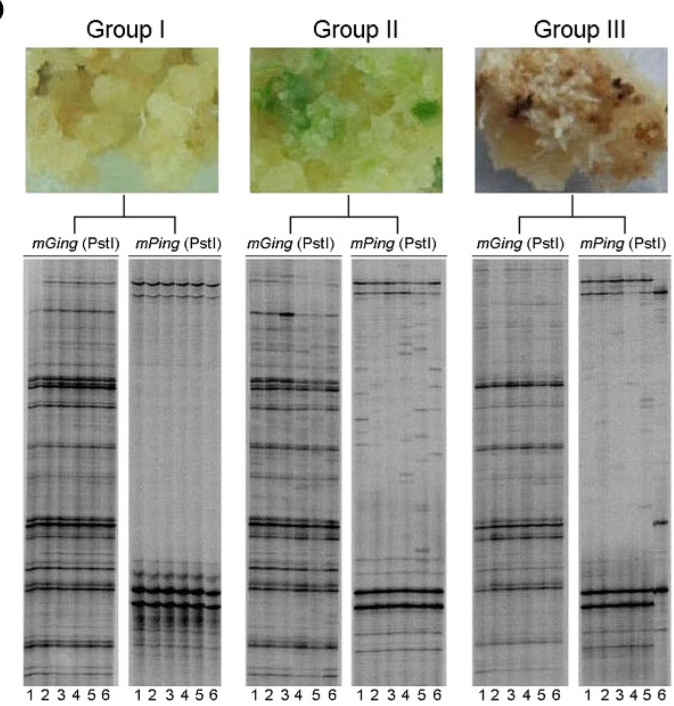

Figure 8 Analyses of transposition frequencies of $m G i n g$ and mPing in successive subcultures and in different culture conditions. (a) Transposition frequencies of $m$ Ging and $m$ Ping in five successive subcultures. One callus from a single plant of Jiahua No. 1 (additional file 6a Group I, lane 1) was selected and divided into four pieces (Group I, II, III, and IV). Each callus piece was cultured for five passages (five tubes). In each passage, part of callus piece was used for gDNA extraction and the rest was transferred into a new tube for the next culture passage. (b) The gDNA sample of each lane in TD gels was from one passage of subculture and the lane number indicated the passage of the subculture.

Autoradiograph of TD gels of $m$ Ging and mPing in different culture conditions. Each group was composed of 6 callus pieces respectively from 6 independent calli. The same lane number in different groups indicated that the callus pieces were originally from the same callus. Group I, calli were inoculated on cell subculture medium and cultured for 28 days. Group II, calli were inoculated on differentiation medium for 28 days. Group III, calli were inoculated on proliferation medium and cultured for 80 days without transferring.

\section{Discussion}

\section{mGing is an active MITE in rice}

mPing was reported as the first active MITE in plant [12-14]. Recently, Yang et al. constructed a co-transposition system in yeast using rice Mariner-like and Stowaway-like elements to demonstrate that the Stowawaylike MITEs could actively transpose [16]. In this study, we showed that mGing, as a new member of Gaijin family, was actively transposing in rice under stress conditions.

Here, the question to be answered is why the number of active MITEs is so rare, although a huge number of MITEs have been identified. There could be two possibilities that account for this phenomenon. Firstly, MITEs might have been very active in the early stage during evolution process, which led to an accumulation of many different MITEs in terms of the numbers and types. For some unknown reasons that are yet to be explicated, the majority of the MITEs became "silenced" and only a small number of MITEs were found to be active nowadays, such as mPing and mGing. However, the current evidence, to our knowledge, did not validate this speculation. It has been suggested that MITEs may originate from autonomous DNA transposons and their transposition was facilitated by the use of transposases encoded from the corresponding autonomous transposons [5,7]. Thus far, there have been no tendencies showing that majority of autonomous DNA transposons were silenced. In contrast, the activities of these autonomous DNA transposons could be detected fairly easily under stress conditions. Thus, it is hard to speculate that the MITEs alone could be silenced independent of other transposon systems.

Secondly, MITEs may have been always active within the genome during evolution, but their movement was difficult to be detected due to the current available techniques. In fact, present methods used for detection of MITE transposition are not many, only including Southern hybridization, TD and co-transposition analysis etc., which all have some limitations. For example, Southern blotting can give clear and reliable indication of the MITE movement, however it lacks sensitivity. Especially for the MITEs with high copy number, it cannot distinguish the banding patterns among different samples. Use of co-transposition analysis could provide direct evidence for MITE transposition and able to confirm crossmobilization between MITEs and the related autonomous elements. However, this is an in vitro assay and may not be able to reflect the real situation of the MITE movement in the genome.

TD is one of the simple and most commonly used methods for MITE detection. The main issue with TD assay is that the polymorphic bands revealed by TD are 
sometimes not easily to confirm by PCR validation in some cases. The reported filler DNA insertion at the insertion sites [14] and our discovery of SNPs introduction within the flanking sequences of the insertion sites after MITE transposition are the possible explanations of the discrepancy between TD bands and PCR validation. In addition, significant size variations of the restriction fragments could also lead to an un-even PCR amplification. Especially for the MITE with high copy numbers ( $>1000$ copies), the detection sensitivity was limited by the number of bands that could be revealed by denaturing PAGE.

In the present study, we replaced tetracutter enzymes that have been used in most of the published studies, such as MseI, with hexacutter restriction enzymes (e.g., PstI), reduced the number of the fragments to be amplified and improved the sensitivity of TD assay. In addition, we employed inverse PCR method to discover more transposition sites. The inverse PCR method helped us to overcome the obstacle that heterozygous excision or heterogeneous excision cannot be revealed in TD assay and aided the discovery of three new transposition sites of $m$ Ging in irradiation condition.

Together, our data and others suggested that MITE transposition may not be a rare event in plant. With further improvements in detection techniques, it is likely that more active MITEs can be found in the different biological processes.

Unique structural features of $m$ Ging and its target regions Two key aspects of the sequence and structural characteristics for MITEs have been well documented [11,16,21]: i) TIRs are integral portions of the transposon and usually complementarily identical in newly transposed copies; ii) MITEs tend to insert into AT-rich regions where a pair of TSD may be generated. In this study, sequence variation of $m$ Ging was firstly observed by base composition analysis of its TIR region. Subsequently, we noted that five newly transposed $m$ Ging copies contained scattered SNPs and any of two copies exhibited at least $90 \%$ homology with each other.

Previous study observed that the element size affected transposition efficiency in non-autonomous elements; transposase binding differed between non-autonomous elements due to the internal sequence variation [16]. Thus, higher level of sequence variation in $m$ Ging family compared with the mPing family might account for the different transposition efficiency between two MITE families. The TIR sequences of five newly transposed copies are not perfectly conserved, but each TIR sequence contains an 8 bp motif "AACTAAAC (the complementary sequence "GTTTAGTT") (See additional file 7: Sequence alignment of the $m$ Ging elements from new transposition sites). We conjectured that this
8 bp motif might be a site which is necessary for $m$ Ging transposition, such as transposase recognition [6].

The surrounding sequences of target sites for both insertion and excision were frequently altered by $m$ Ging transposition. When primers pairs were targeted within the altered sequence, negative or unpredicted bands could occur and led to a low frequency of detectable PCR products in validation experiments. This gave an explanation why the polymorphic bands sometimes could not be confirmed by other techniques, for example, PCR. Therefore, this sequence variation suggests that MITE transposition may not be a simple "insertion" or "excision" process and possibly involves an inaccurate DNA replication mechanism, such as break-induced replication (BIR). BIR is a highly inaccurate cellular process that mimics normal DNA replication in its processivity, rate, and capacity to duplicate hundreds of kilobases, but is initiated at double-strand breaks (DSBs) rather than at replication origins [22]. Thus, this may explain why the flanking region of the newly transposed $m$ Ging copies contained some SNPs in this study.

\section{Association of $m$ Ging transposition with differentiation}

Previous studies reported that TE transposition activity could be induced under either biological or non-biological stress conditions [23]; mPing copy number was increased in three assay systems: long-term cell culture, anther culture, and plants derived from seeds with $\gamma$ irradiation [12-14]. However, the relationship between MITE transposition and associated biological phenotypes remains unclear. A complete tissue culture should involve three main processes: calli formation from dedifferentiated explants, cell proliferation of calli and formation of plantlets through re-differentiation. The published data showed that mPing was actively transposed during the processes of the de-differentiation and proliferation [13], but whether the re-differentiation could induce MITE transposition is ambiguous. In this study, a series of parallel experiments were carried out to examine whether re-differentiation contributes to induction of MITE transposition by comparison of the $m$ Ging/mPing transposition frequency in callus within regenerated plantlets.

In anther culture experiment, we observed that mPing was actively transposing in all plantlet samples and $m$ Ging in about $50 \%$ of the samples, but active transposition was not identified in calli for both elements. The above results suggested a close link between mGing/ mPing transposition and cell re-differentiation, as the process from callus to regenerated plantlet is predominantly driven by re-differentiation. Moreover, the transposition frequency of $m$ Ging/mPing in scutellum-derived callus and plantlet exhibited a similar profile as observed in anther culture, further supporting that $m$ Ging/mPing 
transposition is biologically linked to cell redifferentiation.

In the above experimental systems, the transposition of mGing/mPing was not detected in anther-derived or scutellum-derived calli. We reckoned that it was possibly resulted from the difference in rice material or the experimental conditions. To address these issues, three experiments were conducted in parallel to examine the transposition of $m$ Ging/mPing in calli. (i) Comparison of the calli cultured in subculture medium and those in differentiation medium demonstrated that the active transposition of $m$ Ging/mPing occurred only in differentiated calli, which further confirmed the link between cell differentiation and MITE transposition. (ii) Calli were cultured continuously in subculture medium for an extended period of time until they became browning and covered with roothair like structures, and actively transposing mPing was detected in those calli. This phenomenon was also observed in the scutellum-derived calli that had been cultured for 80 days. These results indicated that active transposition of $m$ Ging/mPing was hardly detected in callus proliferation process unless the callus was cultured under stress condition, such as nutrient-deficiency.

Considering that extreme conditions are not physiological, the exact frequency of $m$ Ging/mPing transposition in normal conditions is yet to be defined. (iii) A series of passages of callus cultures were performed in subculture medium (five of 28-days' cultures with fresh medium replaced at each passage). Both $m$ Ging and mPing were transposingly inactive in all passages. Taken three experiments together, we intend to give a reasonable answer to the question "why was the frequency of $m$ Ging/mPing transposition in plantlets markedly higher than that in calli?" The transposition frequency of $m$ Ging/mPing in newly de-differentiated calli is very low in a relatively long physiological culture process; one of the critical factors that induce MITE transposition is the change of cell physiological status, in which cell differentiation should be the significant one.

\section{Conclusions}

$m$ Ging is actively transposed under $\gamma$-ray irradiation or in cell re-differentiation process in rice. This newly identified active MITE, $m$ Ging, provides new insights into the function and significance of transposons in rice genome. Ongoing functional studies are required to investigate its transposition mechanism and regulation, which will further our understanding of its roles and involvements in different biological processes.

\section{Methods}

\section{Plant materials}

Rice materials, including 15 indica cultivars, 3 javanica cultivars, 12 japonica cultivars and 8 O. rufipogon accessions, were supplied by Germplasm Center of China National Rice Research Institute, Hangzhou, China. The plants of Arabidopsis were obtained from the experimental farm of Zhejiang University Huajiachi Campus.

One japonica cultivar Jiahua No. 1 from Jiaxing Academy of Agricultural Science in China was used for $\gamma$-ray irradiation and tissue culture experiments. Seeds of Jiahua No. 1 were irradiated at different doses in NuclearAgricultural Science Institute of Zhejiang University.

\section{Tissue culture}

For anther culture, anthers were collected from a single sterile spike when the pollens were at the miduninucleated stage, and cultured on one-step culture medium at $25^{\circ} \mathrm{C}$. The medium contained MS macro-elements [24], N6 micro-elements [25] and MS Fe-salts supplemented with $2 \mathrm{mg} / \mathrm{L}$ glycine, $4 \mathrm{mg} / \mathrm{L}$ vitamin B1, 0.5 $\mathrm{mg} / \mathrm{L}$ vitamin $\mathrm{B} 5,0.5 \mathrm{mg} / \mathrm{L}$ vitamin $\mathrm{B} 6,0.01 \mathrm{mg} / \mathrm{L}$ 2.4$\mathrm{D}, 3 \mathrm{mg} / \mathrm{L} \alpha$-naphthalene acetic acid (NAA), $3 \mathrm{mg} / \mathrm{L}$ kinetin, $5 \%$ sucrose and $7 \mathrm{~g} / \mathrm{L}$ agar.

For scutellum-derived embryogenic calli culture, immature seeds were harvested from a single spike, whose surface was sterilized in $70 \%(\mathrm{v} / \mathrm{v})$ ethanol. The excised embryos were placed onto subculture medium and cultured in the dark at $25^{\circ} \mathrm{C}$. The subculture medium was composed of N6 macro-elements, B5 microelements, B5 vitamins, MS Fe-salts supplemented with 2 $\mathrm{mg} / \mathrm{L}$ 2, 4-D, $500 \mathrm{mg} / \mathrm{L}$ Glutamine, $500 \mathrm{mg} / \mathrm{L}$ Pro, 300 $\mathrm{mg} / \mathrm{L}$ casein, $3 \mathrm{mg} / \mathrm{L}$ sucrose and $7 \mathrm{~g} / \mathrm{L}$ agar. The calli with a size of approximate $5 \mathrm{~mm}$ were selected and transferred onto fresh subculture media for further growth. The grown calli were divided into two parts for future culture. One part was transferred onto subculture media, while the other part onto differentiation media. The differentiation medium was composed of N6 macro-elements, B5 micro-elements, B5 vitamins, MS Fe-salts supplemented with $3 \mathrm{mg} / \mathrm{L} \mathrm{BA}, 0.5 \mathrm{mg} / \mathrm{L}$ NAA, $30 \mathrm{mg} / \mathrm{L}$ sucrose and $7 \mathrm{~g} / \mathrm{L}$ agar.

\section{Examination of the transposition frequency of $m G i n g /$ $m$ Ping in calli}

In the first experimental system, a well-grown antherderived callus was selected and divided into 4 pieces. Each piece was cultured in a single tube containing the cell subculture medium and in dark to ensure better conditions for cell proliferation. After 28 days, part of the callus was dissected for gDNA extraction and the rest was subcultured into a new tube with fresh subculture medium for another 28 days. After five successive subculture generations, four callus pieces totally yielded 20 samples. In the second experimental system, six independent anther-derived calli were randomly selected and each was divided into 3 pieces that were respectively 
subjected to three different culturing conditions: group I - culturing on subculture medium for 28 days, group II - culturing on differentiation medium for 28 days, and group III - culturing continuously on subculture medium for 80 days.

\section{Computational analysis}

Three rice genome sequences were downloaded from Beijing Genome Institute (BGI, http://rise.genomics.org. cn/rice/index2.jsp), International Rice Genome Sequencing Project (IRGSP, http://rgp.dna.affrc.go.jp/IRGSP/), and Syngenta http://www.syngenta.com/. The genome sequence of Arabidopsis was downloaded from National Center for Biotechnology Information (NCBI, http:// www.ncbi.nih.gov). For each genome, all sequences were merged together and formatted by formatdb program http://www.ncbi.nih.gov. Indexed database was then searched using $m$ Ging sequence as a query on the local computer with NCBI blast program [26]. A Perl script was used to parse the HSPs in output blast report. HSPs with e-value less than $1 \mathrm{e}-20$ and the length ranging between 90-146 bp were counted for each genome. Bioperl modules including Bio::Seq Bio::SeqIO were used in the scripts [27]. All full length $m$ Ging sequences from Nipponbare genome were parsed and collected. Both sides of the flanking sequences with the length of 500 bp were fetched out by e-utility tools for GC content analysis. A 10 bp sliding window over the 500 bp flanking sequence was chosen to calculate the GC content for each window, and then the average GC content was calculated at the same position of all the fetched sequences. Control sequences were randomly collected from the same rice genome with each one having $500+$ $500 \mathrm{bp}$ in length. An $18 \mathrm{bp}$ sequence was extracted from each end of those mGing elements to analyze the TIR composition. A text file including those short sequences was prepared and used to draw a pictogram on WebLogo 3 website http://weblogo.threeplusone. com/. PCA analysis was conducted as previously described [28]. The TD bands were converted into binary data matrix (0/1 if band was absent/present), which was subjected to a PCA analysis using MATLAB (Version 7, The MathWorks, Inc.).

\section{RNA isolation and RT-PCR}

Total RNAs from rice endosperm were isolated using a protocol based on Bekesiova et al. [29]. Trizol ${ }^{\mathrm{TM}}$ reagent was used for total RNAs extraction from other rice organs in accordance to the manufacturer's instructions (Gibco-BRL). DNase I treatment was used to remove genomic DNAs contamination and total RNAs samples were purified with RNeasy mini kit (QIAGEN).

Reverse transcription was performed on $200 \mathrm{ng}$ RNA sample using a RT-PCR kit (Invitrogen). The RT mixtures were incubated at $42^{\circ} \mathrm{C}$ for $1 \mathrm{hr}$ before carried out PCR by using AS primer pair, which was used in an alternative splicing detection experiment. Thermocycling was performed with a thermocycler (ThermoHybaid), using 35 cycles at $94^{\circ} \mathrm{C}$ for $30 \mathrm{sec}, 58^{\circ} \mathrm{C}$ for $45 \mathrm{sec}$, and $72^{\circ} \mathrm{C}$ for $1 \mathrm{~min}$.

\section{DNA extraction and hybridization}

The gDNA was extracted using cetyltrimethylammonium bromide (CTAB) method [30], with modification of extending the CTAB cleaning step using DNA cleaning column (QIAGEN).

Genomic DNA $(5 \mu \mathrm{g})$ was digested with PstI (TaKaRa). After separation in $0.8 \%(\mathrm{w} / \mathrm{v})$ agarose gels, the DNA was transferred to Hybond-N + membranes (Amersham) and irradiated $\left(60 \mathrm{~mJ} / \mathrm{cm}^{2}\right)$ by UV crosslinker (UVP) before hybridization. Hybridization probes (purified PCR products) were labeled with $\alpha-{ }^{33} \mathrm{P}-\mathrm{dCTP}$ (Amersham) using Prime-a-gene ${ }^{\mathbb{R}}$ Labeling System (Promega). The hybridization was carried out in a buffer (1\% BSA, 1 mM EDTA, 0.25 M NaHPO4, pH 7.2, 7\% SDS) for overnight at $60^{\circ} \mathrm{C}$ in a hybridization oven (ThermoHybaid). Subsequently, the nylon membranes were washed twice at $60^{\circ} \mathrm{C}$ in $2 \times \mathrm{SSC}$ containing $0.1 \% \mathrm{SDS}$ for $20 \mathrm{~min}$ and twice in $0.1 \times$ SSC containing $0.1 \%$ SDS for $20 \mathrm{~min}$. The hybridization signals on the membranes were absorbed by storage phosphor screen (Kodak) for $16 \mathrm{hr}$ and signal intensities were measured by Typhoon 9200 PhosphorImager (Molecular Dynamics).

\section{Transposon display}

The TD was carried as described by Van den Broeck et al. [31] and Casa et al. [32] with following modifications. Each gDNA sample (500 ng) was restricted with 30 units Sau3AI, EcoRI, HindIII, XbaI, SalI, or PstI (TaKaRa) by incubation for $3 \mathrm{hr}$ at $37^{\circ} \mathrm{C}$ in $50 \mu \mathrm{L}$ reaction mix, respectively. After precipitated by isopropanol, cassettes were ligated by adding $1 \times$ ligation buffer, 10 units ligase $(\mathrm{TaKaRa})$ and $500 \mathrm{ng}$ Sau3AI cassettes or 50 ng EcoRI, HindIII, XbaI, SalI, or PstI cassettes in $30 \mu \mathrm{L}$ reaction mix with incubation for $45 \mathrm{~min}$ at $16^{\circ} \mathrm{C}$. Preamplification was performed by using an element-specific primer and an cassette-specific primer $\mathrm{C} 1$ (TaKaRa) in a final $20 \mu \mathrm{L}$ volume with 4 pmol of each primer, 1 unit $L A$ Taq DNA polymerase (TaKaRa), $0.2 \mathrm{mM}$ dNTPs and $1 \times$ PCR buffer. For $m$ Ging TD, the PCR condition was programmed to run 22 cycles at $94^{\circ} \mathrm{C}$ for $30 \mathrm{sec}, 58^{\circ} \mathrm{C}$ for $30 \mathrm{sec}$, and $72^{\circ} \mathrm{C}$ for $60 \mathrm{sec}$. Two $\mu \mathrm{L}$ of the 20-times diluted pre-amplified product was taken as template for hot amplification. Radioactively labeling PCRs were performed using $1.5 \mathrm{mM} \mathrm{MgCl}_{2}, 1 \times \mathrm{PCR}$ buffer, 1 unit LA Taq DNA polymerase, 4 pmol of another element-specific primer and an adaptor-specific primer C2 (TaKaRa), $0.2 \mathrm{mM}$ each of dATP, dGTP and 
dTTP, $0.01 \mathrm{mM}$ dCTP, $1 \mu \mathrm{L}$ of $\left[\alpha_{-}{ }^{33} \mathrm{P}-\mathrm{dCTP}\right](10 \mathrm{mCi} /$ $\mathrm{mL}$, Amersham) and deionized water (to $20 \mu \mathrm{L}$ ). The PCR experimental profile for hot amplification was programmed to run one cycle at $94^{\circ} \mathrm{C}$ for $30 \mathrm{sec}, 68^{\circ} \mathrm{C}$ for $30 \mathrm{sec}$, and $72^{\circ} \mathrm{C}$ for $60 \mathrm{sec}$. The annealing temperature was lowered each cycle with $1^{\circ} \mathrm{C}$ for 12 cycles, and then kept at $56^{\circ} \mathrm{C}$ for another 20 cycles. The $m$ Ging primers for TD were designed based on the consensus sequences acquired from the Repbase and specific for $m$ Ging element (See additional file 8: Summary of primer sequences). The cassettes were from $L A \mathrm{PCR}^{\mathrm{TM}}$ in vitro cloning kit (TaKaRa). Primer sequences and PCR conditions of mPing and $I D-1$ were from Jiang et al. [12]. Radioactive labeled PCR products were electrophoresed in 6\% polyacrylamide/urea gels using Life Technologies S2 apparatus. After samples were electrophoresed (50 W constant) for $3 \mathrm{hr}$, the gel was transferred to Whatman $3 \mathrm{~mm}$ paper, dried on a slab gel dryer, and absorbed by storage phosphor screen (Kodak) for $12 \mathrm{hr}$. The signal intensities were measured by Typhoon 9200 PhosphorImager (Molecular Dynamics).

\section{Inverse PCR}

The pre-amplification products in TD analysis, which were amplified by $m$ Ging TD-specific primer 1 and PstI cassette primer $\mathrm{C} 1$, were used as templates for inverse PCR amplification. The fragments were amplified in a $50 \mu \mathrm{L}$ reaction volume using $m$ Ging $\mathrm{TD}$-specific primer 2 and PstI cassette primer C2. The PCR condition was the same as in the TD except that the dNTP concentrations were at $0.2 \mathrm{mM}$ and no ${ }^{33} \mathrm{P}$-labelled dCTP was added. The final product of $10 \mu \mathrm{L}$ was analyzed in an agarose gel stained with ethidium bromide and the rest $40 \mu \mathrm{L}$ products were purified by QIAquick PCR purification kit (QIAGEN), and ligated with T4 DNA ligase (Promega) into $\mathrm{T}$-vector (Promega) for cloning and sequencing.

\section{Re-amplification and sequence analysis}

For bands recovery, the dried gel was exposed to an $\mathrm{x}$ ray film for $24 \mathrm{hr}$. After developing the $\mathrm{x}$-ray film, the polymorphic bands were located by either marking with a pencil or cutting through the developed x-ray film. The gel slice along with the $3 \mathrm{~mm}$ paper was incubated in $100 \mu \mathrm{L}$ deionized water for $30 \mathrm{~min}$, boiled for $15 \mathrm{~min}$ in a tightly capped eppendorf tube, and the gel materials were pelleted by centrifugation. The DNA fragments were recovered by ethanol precipitation in the presence of $0.3 \mathrm{M} \mathrm{NaOAc}, 5 \mu \mathrm{L}$ of $10 \mathrm{mg} / \mathrm{mL}$ glycogen as a carrier, and suspended in $10 \mu \mathrm{L}$ TE $(10 \mathrm{mM}$ Tirs- $\mathrm{HCl}, \mathrm{pH}$ 8.0, 1 mM EDTA) buffer. The fragments were amplified in a $50 \mu \mathrm{L}$ reaction volume using the same primer pair and PCR conditions as in the TD except that the dNTP concentrations were at $0.2 \mathrm{mM}$ and no isotope was added. The amplicons were electrophoresed in a $1 \%$ agarose gel, purified by a PCR cleaning column (Millipore), and cloned into the pGEM-T-easy vector using the TA cloning system (Promega). Plasmid DNA sequencing of cloned fragments with either M13 forward or reverse primer was carried out using the DYEnamic ET Dye Terminator Cycle Sequencing Kit (Amersham) on a MegaBACE ${ }^{\mathrm{TM}} 1000$ automated sequencer (Molecular Dynamics). Multiple sequence alignments were performed using the online ClustalW program http://www.ebi.ac.uk/clustalw/. All new $m$ Ging transposition sites identified in $\gamma$-ray irradiation experiment and anther culture experiment has been deposited in GenBank. The accession numbers of these transposition sites are as follows: $\mathrm{R}^{+}{ }^{+}$[GenBank: JN016853], R5 [GenBank: JN016854], R9 ${ }^{+}$[GenBank: JN016855], R9 [GenBank: JN016856], R13 ${ }^{+}$[GenBank: JN016857], R13 [GenBank: JN016858], A4 ${ }^{+}$[GenBank: JN016859], A4 [GenBank: JN016860], A6 ${ }^{+}$[GenBank: JN016861], A6 [GenBank: JN016862].

\section{Additional material}

Additional file 1: Sequence homology search for mGing copy numbers. A table listed the sequence homology search analysis for mGing copy numbers in different rice genomes and in Arabidopsis genome.

Additional file 2: Southern blot analysis of $m$ Ging. A figure showed the southern blot analysis of $m$ Ging in different rice genome.

Additional file 3: Polymorphism of locations of $m$ Ging (a) and $m$ Ping (b) in rice genomes. The gDNA samples from various rice cultivars and Arabidopsis were digested with a tetracutter restriction enzyme (Sau3Al) or a hexacutter restriction enzymes (EcoRI, Xbal, HindIII, Sall, or Pstl), and ligated to the corresponding cassettes for TD analysis: lane 1, japonica Nipponbare; lane 2, indica 93-11; lane 3, indica Peiai 645; lane 4, Arabidopsis; lane 5, japonica Jiahua No. 1.

Additional file 4: Sequence alignment of $\mathbf{1 5}$ bands from TD gel. The bands were obtained from additional file 2 (a), Pstl, lane 1 were subjected for sequence analysis. The alignment was generated using the online ClustalW program and the nucleotides conserved across more than $75 \%$ total sequences were highlighted in black. Arrow indicated the TIR, and the TSDs were boxed.

Additional file 5: Principal component analysis clustering of 13 rice cultivars and $\mathbf{1 8}$ wild rice accessions. The TD band data from Figure 4 was used to conduct PCA. The first two principal components, which accounted for $50 \%$ of the variation, were showed in the plot.

Additional file 6: Autoradiograph of TD gels of $m$ Ging (a) and mPing (b) in embryogenic culture. A total of 30 scutellum-derived calli with a size of approximately $5 \mathrm{~mm}$ were selected and transferred onto fresh subculture medium for further culturing. After cultured in the dark for 30 days, a well grown callus was selected and half of it was transferred onto fresh subculture medium for further culturing. The other half of callus and 4 randomly selected calli were used for DNA extraction as samples of Group I. For the half of the callus on the fresh medium after 15 days of proliferation-driven growth, ten pieces of cell clusters were taken and each piece was further divided into two parts. One part was transferred to fresh subculture medium, cultured for 15 days and used for DNA extraction as samples of Group II. Another part (from 10 pieces) was transferred onto the differentiation medium and 22 regenerated plantlets were collected for gDNA extraction as samples of Group III. The restriction enzyme Pstl and Pstl cassette were used for TD analysis. 
Additional file 7: Sequence alignment of the $m$ Ging elements from new transposition sites. The sequence comparison of $m$ Ging elements were from five new transposition loci that were validated in irradiation experiments using ten irradiated seedlings ( $R$ ) and in anther culture experiment using 14 plantlets regenerated from anther-derived calli (A) The alignment was generated using the online ClustalW program and the nucleotides conserved across more than $75 \%$ total sequences were highlighted in black.

Additional file 8: Summary of primer sequences. A table listed the all primer sequences used in this paper.

\section{Abbreviations}

MITE: Miniature inverted repeat transposable element; TE: Transposable element; TD: Transposon display; SNPs: Single nucleotide polymorphisms; bp, Base pair; TIR: Terminal inverted repeats; TSD: Target site duplications; NAA: Naphthalene acetic acid; HSPs: High-scoring segment pairs; CTAB: Cetyltrimethylammonium bromide; PLRRP: Putative leucine-rich repeat protein; gDNAs: Genomic DNAs; BIR: Break-induced replication; DSBs: Double-strand breaks; PCA: Principal component analysis.

\section{Acknowledgements}

The authors would like to thank Dr. Lunquan Sun for his critical reading and valuable suggestions for improvement of the manuscript and Dr. Frederick Luk for his help and contribution in preparing the manuscript. This work was supported in part by grants from the National Natural Science Foundation of China grant 30871329 and Natural Science Foundation of Zhejiang grant Y307099. The funding agencies played no role in study design, in the collection, analysis and interpretation of data; in the writing of the manuscript; or in the decision to submit the manuscript for publication.

\section{Author details}

Institute of Biotechnology, College of Agriculture and Biotechnology, Zhejiang University, Hangzhou 310029, China. ${ }^{2}$ National Center for Rice Improvement, China National Rice Research Institute, Hangzhou 310006, China. ${ }^{3}$ Jiaxing Academy of Agricultural Science, Jiaxing 314016, China. ${ }^{4}$ The Unit of Biocomputation, NICHD/NIH, Bethesda, MD 20892, USA.

\section{Authors' contributions}

HTD, KLZ and DBL conceived of the study and participated in its design, coordination, and interpretation. HTD and LZ carried out TD analysis assay, PCR validation experiment. HGY, FCY, BZM, DZ and JY performed the anther culture and embryogenic culture experiments; JC and XXY were responsible for the sequence identification and comparison analysis. HTD, LZ, KLZ, JC and DBL drafted the manuscript. All authors read and approved the final manuscript.

\section{Competing interests}

The authors declare that they have no competing interests.

Received: 8 October 2011 Accepted: 13 April 2012

Published: 13 April 2012

\section{References}

1. McClintock B: The origin and behavior of mutable loci in maize. Proc Natl Acad Sci USA 1950, 36(6):344-355.

2. Craig NL: Target site selection in transposition. Annu Rev Biochem 1997, 66:437-474.

3. Chang RY, O'Donoughue LS, Bureau TE: Inter-MITE polymorphisms (IMP): a high throughput transposon-based genome mapping and fingerprinting approach. Theor Appl Genet 2001, 102(5):773-781.

4. Wessler SR, Bureau TE, White SE: LTR-retrotransposons and MITEs: important players in the evolution of plant genomes. Curr Opin Genet Dev 1995, 5(6):814-821

5. Feschotte C, Jiang N, Wessler SR: Plant transposable elements: where genetics meets genomics. Nat Rev Genet 2002, 3(5):329-341.

6. Feschotte C, Osterlund MT, Peeler R, Wessler SR: DNA-binding specificity of rice mariner-like transposases and interactions with Stowaway MITEs. Nucleic Acids Res 2005, 33(7):2153-2165.
7. Feschotte C, Mouches C: Evidence that a family of miniature invertedrepeat transposable elements (MITEs) from the Arabidopsis thaliana genome has arisen from a pogo-like DNA transposon. Mol Biol Evol 2000, 17(5):730-737.

8. Zhang X, Jiang N, Feschotte C, Wessler SR: PIF- and Pong-like transposable elements: distribution, evolution and relationship with Tourist-like miniature inverted-repeat transposable elements. Genetics 2004, 166(2):971-986.

9. Oosumi T, Garlick B, Belknap WR: Identification of putative nonautonomous transposable elements associated with several transposon families in Caenorhabditis elegans. J Mol Evol 1996, 43(1):11-18

10. Feschotte C, Swamy L, Wessler SR: Genome-wide analysis of mariner-like transposable elements in rice reveals complex relationships with stowaway miniature inverted repeat transposable elements (MITEs). Genetics 2003, 163(2):747-758.

11. Feschotte $C$, Zhang $X$, Wessler SR: Miniature inverted-repeat transposable elements (MITEs) and their relationship with established DNA transposons. In Mobile DNA II. Edited by: Craig NL, Craigie R, Gellert M, Lambowitz A. Washington: American Society of Microbiology Press; 2002:1147-1158.

12. Jiang N, Bao Z, Zhang X, Hirochika H, Eddy SR, McCouch SR, Wessler SR: An active DNA transposon family in rice. Nature 2003, 421(6919):163-167.

13. Kikuchi K, Terauchi K, Wada M, Hirano HY: The plant MITE mPing is mobilized in another culture. Nature 2003, 421(6919):167-170.

14. Nakazaki T, Okumoto Y, Horibata A, Yamahira S, Teraishi M, Nishida H, Inoue $\mathrm{H}$, Tanisaka T: Mobilization of a transposon in the rice genome. Nature 2003, 421(6919):170-172.

15. Yang G, Zhang F, Hancock CN, Wessler SR: Transposition of the rice miniature inverted repeat transposable element mPing in Arabidopsis thaliana. Proc Natl Acad Sci USA 2007, 104(26):10962-10967.

16. Yang G, Nagel DH, Feschotte C, Hancock CN, Wessler SR: Tuned for transposition: molecular determinants underlying the hyperactivity of a Stowaway MITE. Science 2009, 325(5946):1391-1394.

17. Bureau TE, Ronald PC, Wessler SR: A computer-based systematic survey reveals the predominance of small inverted-repeat elements in wildtype rice genes. Proc Natl Acad Sci USA 1996, 93(16):8524-8529.

18. Wang Z, Burge CB: Splicing regulation: from a parts list of regulatory elements to an integrated splicing code. RNA 2008, 14(5):802-813.

19. Ouyang S, Zhu W, Hamilton J, Lin H, Campbell M, Childs K, ThibaudNissen F, Malek RL, Lee Y, Zheng L, et al: The TIGR Rice Genome Annotation Resource: improvements and new features. Nucleic Acids Res 2007, 35:D883-D887.

20. Kohany O, Gentles AJ, Hankus L, Jurka J: Annotation, submission and screening of repetitive elements in Repbase: RepbaseSubmitter and Censor. BMC Bioinforma 2006, 7:474.

21. Jiang N, Wessler SR: Insertion preference of maize and rice miniature inverted repeat transposable elements as revealed by the analysis of nested elements. Plant Cell 2001, 13(11):2553-2564.

22. Deem A, Keszthelyi A, Blackgrove T, Vayl A, Coffey B, Mathur R, Chabes A, Malkova A: Break-induced replication is highly inaccurate. PLOS Biol 2011, 9(2):e1000594

23. Casacuberta JM, Santiago N: Plant LTR-retrotransposons and MITEs: control of transposition and impact on the evolution of plant genes and genomes. Gene 2003, 311:1-11.

24. Murashige T, Skoog F: A revised medium for rapid growth and bio assays with tobacco tissue cultures. Physiol Plant 1962, 15(3):473-497.

25. Chu CC: Establishment of an efficient medium for anther culture of rice through comparative experiments on the nitrogen sources. Sci Sin 1975, 18:659-668.

26. Altschul SF, Gish W, Miller W, Myers EW, Lipman DJ: Basic local alignment search tool. J Mol Biol 1990, 215(3):403-410.

27. Stajich JE, Block D, Boulez K, Brenner SE, Chervitz SA, Dagdigian C, Fuellen G, Gilbert JG, Korf I, Lapp H, et al: The Bioperl toolkit: Perl modules for the life sciences. Genome Res 2002, 12(10):1611-1618.

28. Jonathon S: A tutorial on principal component analysis. Measurement 2005, 51(10003):52.

29. Bekesiova I, Nap JP, Mlynarova L: Isolation of high quality DNA and RNA from leaves of the Carnivorous plant Drosera rotundifolia. Plant Mol Biol Reporter 1999, 17(3):269-277. 
30. Murray MG, Thompson WF: Rapid isolation of high molecular weight plant DNA. Nucleic Acids Res 1980, 8(19):4321-4325.

31. Van den Broeck D, Maes T, Sauer M, Zethof J, De Keukeleire P, D'Hauw M, Van Montagu M, Gerats T: Transposon display identifies individual transposable elements in high copy number lines. Plant J 1998, 13(1):121-129.

32. Casa AM, Brouwer C, Nagel A, Wang L, Zhang Q, Kresovich S, Wessler SR: The MITE family heartbreaker (Hbr): molecular markers in maize. Proc Natl Acad Sci USA 2000, 97(18):10083-10089.

doi:10.1186/1471-2164-13-135

Cite this article as: Dong et al: A Gaijin-like miniature inverted repeat transposable element is mobilized in rice during cell differentiation. BMC Genomics 2012 13:135.

Submit your next manuscript to BioMed Central and take full advantage of:

- Convenient online submission

- Thorough peer review

- No space constraints or color figure charges

- Immediate publication on acceptance

- Inclusion in PubMed, CAS, Scopus and Google Scholar

- Research which is freely available for redistribution

Submit your manuscript at www.biomedcentral.com/submit 